\title{
Chinesisches Umweltrecht
}

\author{
Von Joachim Schabacker
}

\section{Vorbemerkung}

Da es im folgenden Beitrag hauptsächlich um das chinesische Umweltrecht gehen soll, mag es verwundern, daß der Durchsetzung des Umweltrechts und der Umweltpolitik relativ viel Platz eingeräumt wurde. Eine rein rechtliche Betrachtung indes scheitert an der chinesischen Realität. Für denjenigen, der die Volksrepublik China und ihre rechtlichen, politischen und gesellschaftlichen Rahmenbedingungen nicht kennt, ergäben sich wahrscheinlich viele Mißverständnisse, wollte man die chinesische Umweltrechtslage von dieser Seite unkommentiert lassen: ${ }^{1}$ Trotz anderthalb Jahrzehnten Reformpolitik und beträchtlicher Bemühungen, zumindest im Wirtschafts- und Umweltbereich ein modernes "Rechtssystem" zu schaffen, bleibt die Benutzung dieses Begriffes im Zusammenhang mit China irreführend. Recht in Form von materiellen Gesetzen spielt nicht die ordnende und unabhängige Rolle, die wir ihm zubilligen, sondern dient großteils immer noch der Verbrämung und Sanktionierung politischer Entscheidungen und weicht diesen oft, wenn es ihnen im Weg steht. $^{2}$

Andererseits ist den chinesischen Entscheidungsträgern und in ihrem Gefolge den Rechtsgelehrten zuzubilligen, daß sie erkannt haben, daß "rule of man" vollständig durch "rule of law" ersetzt werden muß, will man ein zusammenhängendes Rechtssystem in unserem Sinne schaffen und von seinen Vorzügen profitieren. Gerade im Bereich des Umweltrechts ist folglich eine lebhafte Debatte entbrannt, wie die Gesetze und ihre Durchsetzung und eben auch das Verhalten der Exekutive geändert werden müssen, um ein Umweltrechtssystem in unserem Sinne errichten zu können, das dem Verfall der Umwelt Einhalt gebieten kann und den Grundstein für "sustainable development" zu legen vermag, Ziele, die mit

Als Beispiel für die Mißverständlichkeit solchen Vorgehens mag Bernhard Prinz, Luftimmissionswirkungen und Immissionsschutz, in: Bernhard Glaeser, Ökologie und Umweltschutz in der VR China, Bochum 1982, S. 223-272, dienen. Im hier vertretenen Sinne jüngst auch Harro von Senger, Einführung in das chinesische Recht, München 1994, S. 8 und, besonders pointiert, S. 96 f.

$\mathrm{Zu}$ diesen grundsätzlichen Fragen, die hier leider nur ansatzweise behandelt werden können und unten unter II.3. näher für den Umweltbereich untersucht werden sollen, ausführlich und differenziert Albert H.Y. Chen, An Introduction to the Legal System of the People's Republic of China, Singapur, Malaysia, Hong Kong 1992. 
dem traditionellen chinesischen System des rule of man und einem von ihm dominierten Recht nicht erreicht wurden und nicht zu erreichen sind.

Im folgenden stellt der Artikel zunächst die Situation der Umwelt dar, um die Dringlichkeit einer Änderung zu illustrieren. Anschließend werden das geltende Umweltrecht und seine Probleme vorgestellt. Im Schlußkapitel sollen die Verknüpfungen zwischen Umweltpolitik und Umweltrecht analysiert und eine Beurteilung der Entwicklung des (weit verstandenen) Umweltrechts bis heute versucht werden. ${ }^{3}$

\section{Die Lage der Umwelt in China}

Chinas Umwelt ${ }^{4}$ befindet sich in einer Lage, die von ausländischen Beobachtern seit den 80er Jahren und von chinesischer Seite neuerdings ebenfalls ${ }^{5}$ als dramatisch und im höchsten Maße gefährlich für das wirtschaftliche Wachstum und die Gesundheit der Bevölkerung beschrieben wird. Umweltexperten schätzen, daß China mittlerweile mehr als das jährliche Wachstum seines Bruttosozialprodukts von $13 \%$ durch Umweltschäden verliert. ${ }^{6}$

Ein Grụnd für den Niedergang der Umwelt ist Chinas ungünstige Ausgangslage: Als Land mit der größten Bevölkerung der Erde verfügt es pro Kopf bei keinem Rohstoff und keinen Naturressourcen über mehr als $50 \%$ des Weltdurchschnitts ${ }^{7}$, meistens liegen die Werte

Vgl. zum Ganzen Michael Kloepfer / Sigrid Reinert, Aspekte des Umweltrechts in der DDR, in: ZfU 1/90, S. 1. Es zeigen sich erstaunliche Parallelen zu allen hier konstatierten Entwicklungen, Problemen und Defiziten.

4

Vgl. zum folgenden die Oktober 1991-Ausgabe von "das neue China" mit dem Schwerpunktthema Umwelt und vor allem Dirk Betke, Die Umweltfrage, in: Erhard Louven (Hrsg.), Chinas Wirtschaft zu Beginn der 90er Jahre, Hamburg 1989, S. 54-82. Trotz der etwas veralteten Angaben liegt hier die immer noch lesenswerteste Zusammenfassung in deutscher Sprache vor. Der Verfasser dankt Uli Bartel für diesen Hinweis. Neueste Zahlen enthält der 1994 Report on the State of the Environment in China, in: China Environment News, Juni 1995, S. 4-7 (voller Text).

Noch nach dem Massaker auf dem Tianan Men 1989 waren warnende Bücher chinesischer Autoren verboten worden (Vaclav Smil, A Land Stretching to Support Its People, in: International Herald Tribune, 30.5.1994, S. 8). In Chinas Agenda 21 wird dagegen der verheerende Zustand der Umwelt gezielt als Argument eingesetzt, um für China ausländische Hilfe zur Rettung der Umwelt zu fordern. Inzwischen finden sich auch in der Tagespresse regelmäßig Artikel, die sich kritisch mit dem Zustand der Umwelt auseinandersetzen. Vgl. z.B. Zhong Minyuan / Miao Xiaobo, Lagging Environmental Protection Troubles Fujian in Economic Growth, in: China Environmental Joumal, 29.3.1994.

Vaclav Smil, Fn. 5, S. 8.

Diese Angaben und die folgenden Zahlen finden sich z.B. in China's Agenda 21 - white paper on China's population, environment, and development in the $21^{\text {st }}$ century, Beijing 1994, Abschnitt 14, S. 136 ff., und bei Vaclav Smil, Fn. 5, S. 8; Dadi Zhou, Environmental Considerations in the 
darunter: so für Süßwasser (<30\%), Ackerland (25\%), Wald (14,3\%) und Mineralien $(<50 \%)$. Andererseits hat sich die chinesische Regierung vorgenommen, China zu einem entwickelten Land zu machen und nach der Phase der sozialistischen Industrialisierung nunmehr die der marktwirtschaftlichen Revolution folgen zu lassen. Die Folgen sind explosionsartige Vermehrung der ländlichen Kleinindustrie ${ }^{8}$, kräftiges Wachstum der Industrie in den Städten und nahezu ungebremste Umweltverschmutzung im Namen des wirtschaftlichen Fortschritts. Sollte China sich wirklich auch nur annähernd dem Wohlstandsniveau westlicher Industrienationen nähern wollen, kann es das weder mit seinen eigenen Ressourcen erreichen noch fehlende auf dem Weltmarkt hinzukaufen ${ }^{9}$, zumal die Bevölkerung weiter wächst ${ }^{10}$. Seinem Wachstum sind natürliche Grenzen gesetzt, an die es zumindest in Hinblick auf die Umwelt heute schon zu stoßen droht. So wundert es nicht, daß es im autoritären China zu unorganisierten lokalen Erhebungen gegen einzelne Umweltverschmutzer gekommen ist und immer wieder Wissenschaftler und hohe Beamte vor den Folgen der ungezügelten Umweltausbeutung warnen. ${ }^{11}$

Die Städte entlassen $95 \%$ ihrer Abwässer ohne jegliche Klärung in Flüsse und das Meer, $90 \%$ aller Städte beziehen ihr Trinkwasser aus verseuchten Quellen, 65 \% der Bevölkerung sind von trinkbarem Wasser abgeschnitten, 150 Mio. Menschen trinken verseuchtes Wasser. Gleichzeitig steigt der Bedarf an Wasser ständig; Industrie, Landwirtschaft, Städte und Gemeinden konkurrieren um die knappe Ressource. Der Grundwasserspiegel sinkt stetig, weite Landstriche versalzen oder vertrocknen, Flüsse und Küstengewässer sind stark verseucht und leergefischt. ${ }^{12}$

Economic Development of China, in: Arizona Journal of International and Comparative Law, Vol. 9 (1992), No. 1, S. 221, 225-228. Allerdings weichen Zahlenangaben in China auch von Amts wegen bisweilen erheblich voneinander ab, je nach Herausgeber und Zweck der Veröffentlichung. An der tendenziellen Richtigkeit der hier benutzten Angaben besteht indes wenig Zweifel. - Einzige Ausnahme bei den knappen Ressourcen ist Kohle: Die Vorräte reichen für mindestens 1000 Jahre, vgl. Zhao Baoxing, Grave Pollution at Shanxi, Shaanxi and Inner Mongolia Coal Production Base, in: China Science, 15.8.1994.

8 Die Umweltverschmutzung durch die ländliche Kleinindustrie nimmt rasch zu: in den letzten 5 Jahren Abwässer $+65,4 \%$, Staub $+23,4 \%$, Abf alle $+56,9 \%$, Tendenz steigend; vgl. Umweltreport 1994, oben Fn. 4.

9 Es ist nicht denkbar, daß die dazu nötigen Mengen an Ressourcen jemals vom Weltmarkt zur Verfügung gestellt werden können, ganz davon zu schweigen, ob China sie jemals bezahlen könnte (so Vaclav Smil, Fn. 5, S. 8, und Fritz Vorholz, Fluch der großen Zahl, in: Die Zeit, 17.6.1994, S. 33).

Derzeit um etwa 13 Mio. p.a. Vgl. dazu Xiong Zhihong, The Number of China's Population to Approach the Limit of the Environment Bearing Capacity, in: China Environment News, 21.1.1995.

Umweltkatastrophe droht, in: China aktuell, Februar 1989, S. 86.

12

China's Agenda 21, neben dem oben (Fn. 7) genannten Kapitel 14 finden sich Angaben zu den jeweils behandelten Themen auch in allen anderen Kapiteln; Umweltkatastrophe droht, in: China aktuell, Februar 1989, S. 86; Guo Yalin / Zhang Xuejian, Chinese Minister Calls for Effective 
Das knappe Ackerland schrumpft durch Erosion, Wachstum der ländlichen Industrie und Verstädterung. Seit den 50er Jahren hat China ein Drittel seiner jetzigen Ackerflächen verloren, 35 Mio. ha durch Überbauung und über 10 Mio. ha durch Erosion und Vorrücken der Wüste. Exzessive Bewässerung, Düngung und Schädlingsbekämpfung sowie Müllagerung verseuchen mindestens $10 \%$ der verbleibenden Flächen. ${ }^{13}$ In vielen großen Städten sinkt der Boden ab durch unkontrolliertes Fördern von Grundwasser. ${ }^{14}$

Auf 13,4\% der Fläche Chinas wächst heute noch Wald; seine Qualität ist schlecht, die reifen Wälder werden 1997 abgeholzt sein. Die wachsende ländliche Bevölkerung mit ständigem Bedarf an Brennholz macht Aufforstungsbemühungen zunichte ${ }^{15}$, die Überlebensrate aufgeforsteter Bestände beträgt nicht einmal ein Drittel. ${ }^{16}$ Chinas ehemals reiche Grasländer sind durch Übergrasung und falsche Nutzung zu einem Drittel alkalisch oder sandig geworden, ein weiteres Drittel leidet unter Ratten- und Insektenplage. Die Grasproduktion beträgt trotz günstiger klimatischer Bodenbedingungen nur 5-10\% des Ertrages in den USA oder Australien. ${ }^{17}$

China bezieht $95 \%$ seiner Energie und $80 \%$ der industriellen Rohmaterialien aus Mineralressourcen. Die Effizienzwerte der Mineralnutzung liegen aber bei zwei Drittel bis drei

Protection of Water Ressources, in: Information Weekly, 25.3.1994; Zhao Guanliang, China Faces Problems in Water Quality and Quantity, in: China Environment Journal, 17.5.1994; Li Xiaomin, World Bank Presents Report on Management of Chinese Cities, China Construction, 12.11.1994. Um den industriell bedingten Wassermangel Beijings zu lindern, wird nun ein $1.235 \mathrm{~km}$ langer Süd-Nord-Kanal geplant, dessen ökologische Folgen nicht absehbar sind, vgl. Jing Gui, Beijing's Project of Diverting Water from South to North China, in: Beijing Daily, 3.4.1994. Zur Situation der Küstengewässer vgl. Lin Ying, Ecological Environment of Chinese 13 Coastal Waters Worrying, in: Guangming Daily, 6.4.1994.

3 Umweltprobleme, in:China aktuell, Juli 1989, S. 486; Umweltverschmutzung, in: China aktuell, Mai 1989, S. 329; Fritz Vorholz, Fn. 9, S. 33; China's Agenda 21, Fn. 7; Eco-Agriculture Becomes only Choice for China, in: China Environment Journal, 21.4.1994; Zhao Lianqing, Land Resources in China Call for Protection, in: China Industries Journal, 6.6.1994; Zou Zijin, Land Area Shrinks Drastically, Ecological Environment Deteriorates in China, in: Economic Information Daily, 27.7.1994. Siehe zu neuerdings geäußerten Vermutungen, die tatsächliche Ackerfläche Chinas sei um 25 Mio. ha größer als bisher angenommen (statt 95 Mio. 120 Mio ha.): Survey Underestimated Farmland, in: China Daily, 10.3.1995.

Vgl. z.B. She Mengxin, China's Suzhou, Wuxi and Changzhou Suffer Serious Land Subsidence, in: Guangming Daily, 26.4.1994. schen Botschaft Peking.

Waldbestände nehmen ab, in: China aktuell, Januar 1990, S. 17; The Editorial Board of "China Conservation Strategy", China Conservation Strategy, Beijing 1990, S. 31 ff.; China's Agenda 21, Fn. 7.

17 Graslandverluste alarmierend, in: China aktuell, Juni 1989, S. 419; China's Agenda 21, Fn. 7; Weng Hao, Grassland Degeneration in Inner Mongolia Worrying, in: China Environment Journal, 6.8.1994. 
Viertel unter denen der Industrienationen. China verbraucht pro Kopf (!) 2-4 mal mehr Mineralien als die Industrieländer. ${ }^{18}$ Gleichzeitig werden bei der meist unkontrollierten Förderung ganze Landstriche verwüstet und unbrauchbar gemacht, Rekultivierung findet nicht statt.

Die Luftqualität in Chinas Städten ${ }^{20}$ gilt als die schlechteste Asiens, kleinste Partikel, $\mathrm{SO}_{2}$ (10-15 mal mehr als WHO-Grenzwerte) und Stickoxide lassen luftverschmutzungsbezogene Atemwegserkrankungen zur häufigsten Todesursache werden. Nicht mehr nur im Süden des Landes nimmt die Menge des sauren Regens jährlich um mehr als $10 \% \mathrm{zu}$. Das liegt daran, daß $78 \%$ der landesweit produzierten Energie aus Kohle stammen, der technische Stand der Industrie extrem veraltet ist (mit Effizienzraten $30 \%$ unter denen der Industrienationen) und die Anzahl der Kraftfahrzeuge ständig wächst (im Jahr 2000 sollen es 50 Mio. sein; 1994: 8 Mio.). Sie stoßen 15-20 mal mehr Schadstoffe aus als die der Industrienationen. ${ }^{21}$ Zwei Drittel der städtischen Bevölkerung sind Lärm ausgesetzt, der die zulässigen Standards bei weitem übersteigt. ${ }^{22}$

Das gilt v.a. für Energiegewinnung: z.B. Joseph R. Profaizer, Economic Development and Environmental Law in China's Special Economic Zones, in: Texas International Law Journal, Vol. 28, No. 2, 1993, S. 320, 327.

China's Agenda 21, Fn. 7, S. 165 f. Die Tagespresse berichtet wöchentlich über Mineralien-, vor allem Goldräusche, tausende Glücksritter ziehen in vermeintlich erfolgversprechende Gebiete und hinterlassen verwüstete und vergiftete Landstriche. Vgl. z.B. Xu Guozhu, Gold Production in Tongguan Results in Grave Pollution, in: Worker's Daily, 13.7.1994; Wang Jikun Zhen Jian, Illegal Gold Production in Shandong Province Causes Mercury Poisoning, in: China Environment Journal, 28.7.1994; Jin Fengming, Wanton Gold Digging Causes Serious Poisoning of River in Hunan, in: China Environment Journal, 23.6.1994; Gan Qunyan, Sulfur Kilns Pose Threat to People in Shandong, in: China Environment Journal, 2.6.1994; Yu Donghui, Mountain People in Hunan, Guizhou Produce Arsenic by Indigenous Methods, in: China News Service, 11.7.1994.

Zum Zusammenhang zwischen wirtschaftlichem Wachstum und Umweltverschmutzung in großen Städten i.a. und in Chinas Sonderwirtschaftszonen sowie zu deren besonderer Lage im hier interessierenden Zusammenhang s. Joseph R. Profaizer, Fn. 18, S. 320, 324 f. m. Fn. 33; vgl. i.ü. Umweltreport 1994, oben Fn. 4, S. 4 f.

China's Agenda 21, Fn. 7, S. 202 ff.; Priority Programme for China's Agenda 21, Beijing 1994, 4-4-1; Fritz Vorholz, Fn. 9, S. 33; Verschmutzungsgrad bedrohlich, in: China aktuell, Mai 1990, S. 346; aus der chinesischen Tagespresse z.B. Xu Guoying, Hazardous Matter in Vehicle Emissions Exceeds State Standards in Shanghai, in: Wen Hui Bao, 22.3.1994; Huang Yihua, Acid Rain, a Serious Problem for Shenzhen, in: Shenzhen Special Zone News, 8.2.1992; Chen Haibo, China and Japan Make Joint Efforts in Find out the Cause of Acid Rain, in: China Environmental News, 28.7.1994. $\mathrm{Zu}$ angeblich bahnbrechenden Verbesserungen bei Förderung und Aufbereitung von Kohle in jüngster Zeit vgl. Yang Lihua, Chinese Coal Industry Makes Remarkable Results in Environmental Protection, in: China Environment Journal, 10.4.1994. Zusammenfassend zum Problem des sauren Regens Zhong Bian, China's Acid Rain Damage Presents Queckened Tendency, in: China Environmental News, 14.1.1995.

22 Zhao Xiao, Acoustic Environment Not Good in Most Chinese Cities, in: China Environment Journal, 6.12.1994. 
Außerdem droht China im Müll zu ersticken: 600 Mio. $t$ industriellen und über 130 Mio. $t$ städtischen Mülls kommen jährlich zu den sich heute schon auf 557 Mio. m Ackerland türmenden 6,5 Mrd.t (nur industrieller Abfall) dazu, die städtische Müllmenge wächst jährlich um $10 \%$. Die Industrie nutzt nur $40 \%$ der in ihren Abfällen vorhandenen Rohstoffe. Nur 28,3\% des städtischen Mülls werden sicher gelagert, in den Großstädten sind es sogar nur $2 \%$; der größte Teil wird einfach in die Natur abgeladen und verursacht regelmäßig Unfälle und Umweltverschmutzung (bereits 1991 waren 20.000 ha Fläche auf diese Weise verseucht). Giftige und gefährliche Abfälle werden nicht sicher gelagert, keine der Spezialdeponien erreicht chinesische Umweltsicherheitsstandards. ${ }^{23}$

Schließlich hat China trotz intensiver Schutzbemühungen durch Umweltverschmutzung, Vorrücken von Industrie und Siedlungen und profitable Wilderei bereits etliche Pflanzenund Tierarten verloren, viele weitere stehen am Rande des Aussterbens. ${ }^{24}$

\section{Das materielle Umweltrecht und die Probleme in der Rechtswirklichkeit}

\section{Geschichte des Umweltrechts in China ${ }^{25}$}

Trotz einiger Versuche von chinesischer Seite, in frühen Dynastien bereits Ansätze für Umweltschutzgesetzgebung zu finden ${ }^{26}$, und verstreuter Gesetzgebung im Laufe der ersten

China's Agenda 21, Fn. 7, S. 214 ff.; Priority Programme for China's Agenda 21, Fn. 21, S. 6-5; David Green / Sandra Yip, Environmental Crisis Looming, in: Asia Law, November 1993, S. 20, 26 f. Jüngst wurde ein Register für industrielle Abfälle eingeführt: Liu Xiaojun, Solid Waste Register System Carry out in China, in: China Environmental News, 4.2.1995.

Dazu finden sich fast täglich Berichte in der Presse. Vgl. z.B. Wang Lei, Red Deer on Brink of Extinction in Tarim, in: China Environment Journal, 3.3.1994; Wildlife Conservation, in: Guangming Daily, März 1994; Guo Huan, Gang of Smugglers of Rare Butterfly Specimens Busted, in: China Environment Journal, 11.6.1995; Yu Ping, More Than Half Restaurants in Changchun Serve Wild Animal Meat, in: China Environment Journal, 26.7.1994 etc.

Beiträge zu diesem Thema z.B. in: Bryan Bachner, Sweep before Your Own Door: The Legal Concept of Environmentalism in the Pearl River Delta, in: Ray' Wacks, Hong Kong, China and 1997: Essays in Legal Theory, Hong Kong 1993, S. 229 ff.; David Green / Sandra Yip, Fn. 23, S. 20 ff.; Frank Münzel, Neues Umweltschutzrecht in der Volksrepublik China, in: WGO - MfOR 1979, S. 213 ff.; Bernhard Prinz., Fn. 1, S. 223 ff.; Qu Geping / Li Jinchan, Environmental Management in China, in: UNA SYLVA, Vol. 33, No. 134 (1981), S. 2 ff. und Oskar Weggel, Gesetzgebung und Rechtsprechung im nachmaoistischen China, Teil IX: Das Öffentliche Recht Umweltschutzrecht, in: China aktuell, Juli 1987, S. 575 ff.

Darstellung und Nachweise bei Oskar Weggel, Fn. 25, S. 575, 577; Weggel spricht davon, daß es sich nur um Umweltgesetze, nicht aber um Umweltschutzgesetze gehandelt habe. Ob diese Unterscheidung sinnvoll ist, mag dahinstehen. Ein Umweltrechtssystem gab es jedenfalls nicht. 
beiden Jahrzehnte der Geschichte der Volksrepublik ${ }^{27}$ wird als Beginn des modernen Umweltrechts allgemein die Stockholmer UNO-Umweltschutzkonferenz 1972 angesehen, in deren Folge die chinesische Führung nach und nach selbst ein Umweltbewußtsein ausbildete, um dies dann jeweils in die Form (materieller) Gesetze zu gießen. Höhepunkt dieser Entwicklung war die Verabschiedung des (vorläufigen) Umweltschutzgesetzes 1979, in dem bereits ein Großteil der heutigen Umweltrechtsstruktur angelegt war. ${ }^{28}$ Letztlich blieb aber wegen des Primats der Ökonomie vor allem die administrative Ausformung des Systems schwach und unstrukturiert, das Recht selbst ein Torso. Erst mit dem klaren Bekenntnis zum Umweltschutz in einem Beschluß des Staatsrates vom Februar $1981^{29}$ wurde der Grundstein für das jetzt geltende Umweltrecht gelegt, und in den 80er Jahren entstanden neben einer neuen Verfassung (erstmals mit Vorschriften zugunsten der Umwelt $^{30}$ zahlreiche Gesetze zur Ausformung des Umweltrechtssystems und parallel dazu eine differenzierte Administration. ${ }^{31}$

Bisheriger Höhepunkt dieser zweiten Umweltrechtsphase war die endgültige Bekanntmachung des (verbesserten) Umweltschutzgesetzes im Dezember 1989. ${ }^{32}$

\section{2. Überblick über das Umweltrechts- und -verwaltungssystem}

\section{a. Bestimmungen des materiellen Umweltrechts}

Der folgende Abschnitt stellt die wichtigsten Charakteristika des geltenden Umweltrechtssystems vor. Dabei wird von einem materiellen Gesetzesbegriff ausgegangen, der alle Formen von Recht umfaßt, die faktisch Gesetzeskraft besitzen (dies können in China neben

Vor allem im Bereich von Arbeitssicherheit/Hygiene und Atomreaktorsicherheit, s. zu dieser Zeit auch Frank Münzel, Fn. 25, S. 213 ff.

Ausführlich dazu Oskar Weggel, Fn. 25, S. 575, 577 ff. Vgl. auch Kaminski in Otto Kimminich / Heinrich Freiherr von Lersner / Peter-Christoph Storm (Hrsg.), Handbuch des Umweltrechts, Bd. I, 2. Auflage 1994, Spalte 359, 359-365.

"Beschluß über die Verstärkung der Umweltschutzarbeit während der Regulierungsperiode der Volkswirtschaft", Nachweis bei Oskar Weggel, Fn. 25, S. 575, 579. Bryan Bachner, Fn. 25, S. 229, 247 ff.

Dieses und alle im folgenden zitierten Umweltgesetze sind in chinesischer Sprache veröffentlicht in: Sammelband von Gesetzen und Regeln zum Umweltschutz (Huanjong baohu fagui huipian), Band I 1989 und Band II 1993, erschienen beim Chinesischen Umweltwissenschaftsverlag (Zhongguo huanging kexue chuban she chuban); Das Umweltschutzgesetz in englischer Sprache ist z.B. 1992: bei demselben Verlag erschienen. 
Rechtsverordnungen z.B. auch interne Weisungen an Parteifunktionäre sein, sogenannte $n e i b u$, die der Öffentlichkeit in der Regel nicht zugänglich sind). ${ }^{33}$

\section{aa. Überblick}

Die Verfassung schreibt die Verantwortung des Staates für Erhalt und Schutz der Umwelt in den Art. 9 II, $10 \mathrm{~V}$ und 26 fest. Unterhalb der Verfassungsebene folgt als "Basisgesetz" das Umweltschutzgesetz, dem wiederum einzelne "spezifische Gesetze" untergeordnet sind. ${ }^{34}$ Auf der nächsten Ebene sind ca. 20 "Regularien" angesiedelt, die eher technischen Charakter haben und der Implementierung der allgemeineren Gesetze dienen. Schließlich folgen über 300 Methoden und Standards. Abgeschlossen wird das Gesetzessystem durch Provinz- und lokale Gesetzgebung, die im Umweltbereich nur in Ermangelung gesamtstaatlicher Gesetze oder in Einzelfällen zur Verschärfung bestimmter Schutzstandards zulässig sind, jedoch gemessen an ihrer Zahl ständig an Bedeutung zunehmen. ${ }^{35}$

Die Volksrepublik China hat mehr als 20 Verträge des Völkerrechts im Umweltschutzbereich unterzeichnet, von denen aber bisher erst wenige in nationale Gesetzgebung umgesetzt worden sind. ${ }^{36}$

\section{bb. Prinzipien des Umweltrechts}

Von chinesischer wie von ausländischer Seite sind im Umweltrecht der Volksrepublik unstreitig folgende drei Prinzipien ausgemacht worden: das Prinzip der harmonischen Entwicklung, das Präventionsprinzip und das Verschmutzer-zahlt-Prinzip.

(1) Mit dem Prinzip der harmonischen Entwicklung ${ }^{37}$ sind die gleichzeitige Entwicklung von Wirtschaft, Gesellschaft und der Erhalt bzw. die Verbesserung der Umwelt zu gegen-

Siehe zur schwierigen Frage der Benennung chinesischer Vorschriften und der damit verbundenen Bedeutungsunterschiede Hsia Tao-Tai, Law Making in The People's Republic of China: Terms, Procedures, Hierarchy, and Interpretation, Washington, D.C., 1986.

Es sind dies das Gesetz zur Vorbeugung und Kontrolle von Wasserverschmutzung, das Gesetz zur Vorbeugung und Kontrolle von Luftverschmutzung, das Gesetz zum Schutz der Meeresumwelt und das Gesetz zum Erhalt wildlebender Tiere. Daneben gibt es eine Reihe anderer Gesetze, die in ihrem Bereich selbst Basisgesetz sind, aber im Bereich des Umweltschutzes zum Umweltschutzgesetz als Spezifisches Gesetz gelten, z.B. die Gesetze für Wald, Grasland, Wasser, Mineral-Ressourcen, Fischerei, Landmanagement.

5 Siehe z.B. die Berichterstattung in China Environmental News, oder ausführlicher die chinesische Ausgabe zhongguo huanjing bao. Priority Programme for China's Agenda 21, Fn. 21, 1-1 A-1.

7 Inwieweit dieses Prinzip über bloße Absichtserklärungen hinaus tatsächlich Eingang in das Umweltrecht gefunden hat, wird noch zu untersuchen sein. 
seitigem Nutzen gemeint. Umwelt und Wirtschaft seien eine dialektische Einheit, deren Gegensatz durch die Implementierung korrekter Pläne und Vorschriften ${ }^{38}$ überwunden werden könne. So werden übrigens auch der gleichzeitige Schutz der Umwelt und der der sozialistischen Modernisierung in Art. 1 Umweltschutzgesetz gedeutet. ${ }^{39}$ Nichts anderes als dieses Prinzip ist schließlich gemeint, wenn in letzter Zeit vor allem im Zusammenhang mit "China's Agenda 21" häufig von "sustainable development" die Rede ist. ${ }^{40}$

(2) In Umsetzung des Präventionsprinzips kennt das chinesische Recht Environmental Impact Assessments (EIA), die sogenannten "drei simultanen Schritte" und integrierte Raumplanung, die im folgenden näher dargestellt werden sollen. Ziel ist es, statt kostenauf wendiger Schadensbeseitigung nach Umweltschädigungen von vornherein Schäden so gering wie möglich zu halten durch Veränderung der Produktionsprozesse, Kontrolle der Produktionsabfälle und Regelung der Verantwortung. ${ }^{41}$

(3) Das Verschmutzer-zahlt-Prinzip ist nicht zuletzt Ausdruck des traditionellen chinesischen Fairneßdenkens. ${ }^{42} 7 \%$ der Investitionen müssen bei neuen Projekten wie Bauvorhaben für Umweltschutz ausgegeben werden, Emissionen über Standards hinaus kosten Gebühren oder Strafen, es existiert ein umfangreiches Haftungssystem für die Verursachung von Umweltschäden. Außerdem experimentiert das China im Umbruch von der Plan- zur Marktwirtschaft mit ökonomischen Anreizen, um über Kosten gewünschtes Verhalten anzuregen.

\section{cc. Einzelne Aspekte des Umweltrechts}

Im nun folgenden Abschnitt werden die wichtigsten Teilaspekte des materiellen Rechts dargestellt. $^{43}$

Bzw. policies, Zhang Kunmin / Jin Ruilin, A Course in China's Environmental Protection Law, Beijing 1992, S. 42.

China's Agenda 21, Fn. 7; Priority Programme for China's Agenda 21, Fn. 21, S. 1 ff.; Future needs growth, environment, in: China Daily, 8.7.1994, S. 1; Green plan receives overseas support, in: China Daily, 24.6.1994, S. 1.

41

Zhang Kunmin / Jin Ruilin, Fn. 38, S. 51 ff, 107 ff; Qu Geping, China's Environmental Policy and World Environmental Problems, in: International Environmental Affairs, Vol. 2, No. 2 (Spring 1990), S. 103, $105 \mathrm{ff}$.

42

Lester Ross / Mitchell Silk, Environmental Law and Policy in the People's Republic of China, New York, Westport (Connecticut), London 1987, S. 208 f.

43

Vgl. zum folgenden z.B. Wang An / Bai Zhengzhu / Yan Junpian, Umweltschutz und Gesetz (Huan jing bao hu yu fa), Beijing 1994, S. 43 ff., 61 ff. 


\section{(1) EIA und drei simultane Schritte}

Sinn einer EIA ist es, bereits vor Konstruktionsbeginn die Umweltauswirkungen eines Projekts vorherzusehen und Umweltüberlegungen in die Planung mit einzubeziehen. Der Durchführbarkeitsstudie (feasibility study) für ein Projekt muß die EIA beigelegt werden (Art. 13 UmweltschutzG), in der kurz- und langfristige Auswirkungen auf die umliegenden und die entfernteren Umweltmedien darzustellen und die Umweltschutzmaßnahmen, die die vorliegende Einheit zu ergreif en plant, vorzustellen sind. Letztere müssen bei geplanten Emissionen Meßeinrichtungen und Datenerfassung umfassen. Sind Umweltverschmutzungen geplant, muß eine Wirtschaftlichkeitsanalyse der Umweltauswirkungen angefertigt werden. Die zuständige Umweltschutzbehörde hat die EIA innerhalb von zwei Monaten zu prüfen. Lehnt sie sie ab, so darf das Projekt nicht genehmigt werden. Wird es dennoch durchgeführt, so ist die Umweltschutzbehörde berechtigt, seinen Betrieb zu suspendieren (Art. 36 UmweltschutzG) ${ }^{44}$

In eine ähnliche Richtung gegen die drei simultanen Schritte: Es sollen bei Planung, Errichtung und Betrieb eines Projektes immer gleichzeitig Umweltschutzmaßnahmen geplant, errichtet und betrieben werden (Art. 26 UmweltschutzG).

\section{(2) Verbot des Transfers von Umweltverschmutzung}

Dieses Verbot richtet sich einerseits gegen den Import umweltschädlicher Produkte oder Techniken aus dem Ausland ${ }^{45}$, sofern keine geeigneten Schutzmaßnahmen zur Verfügung stehen (Art. 30 UmweltschutzG), andererseits aber auch gegen den Transfer umweltschädlicher Produktion innerhalb Chinas, hier vor allem aus den Städten in ländliche Gegenden (Art. 34 UmweltschutzG). Es ist z.B. explizit verboten, 20 bestimmte Technologien an Betriebe der ländlichen Kleinindustrie zu verkaufen. ${ }^{46}$

\section{(3) Recycling und integrierte Nutzung}

Wichtig im Zusammenhang mit Prävention sind Überlegungen zur Wiederverwertung benutzter Produkte und Mehrfachnutzung bestimmter Stoffe bzw. vollständigen Ausnut-

44

Sehr ausführlich und sorgfältig stellt die EIA jetzt dar: Zhou Danmin, Umweltschutzverträglichkeitsprüfung in der VR China, Berlin 1994. Der Verfasser dankt Uli Bartel für diesen Hinweis.

45 Vgl. dazu den aufsehenerregenden Fall einer koreanischen Ladung Giftmülls nach Nanjing: Tang Jian, China Tightens the Control of Pernicious Waste from Overseas, in: People's Daily Overseas Edition, 13.6.1994.

46

Regulation 135, Nachweis bei $R$. Nick Anderson u.a., China Environmental Strategy Paper (zwei Bände), Washington World Bank, April 1992, seit 7/1994 offizielles Dokument, Annex 1, S. 11, Nr. 42. 
zung von Rohstoffen geworden. Derzeit läuft in 47 Städten ein Versuch, einen Recyclingmarkt mit Hilfe von verkäuflichen Müllquoten zu errichten. ${ }^{47}$ Im Umweltschutzgesetz sind für Wiederverwertungsaktivitäten Steuer- und Gewinnvorteile vorgesehen (Art. 18, 31). So gefördert, entwickelt sich rasch ein neuer Industriezweig, der Recycling und Umweltschutz betreibt.

\section{(4) Raumplanung}

In vielen Vorschriften weist das Umweltschutzgesetz auf die Bedeutung des Umweltschutzes vor allem für die Stadtplanung hin. ${ }^{49}$ Städte sollen integriert geplant werden, d.h. eine Vielzahl von Zielen soll mit rationalem Layout erreicht werden können. Wichtigster Einzelpunkt in diesem Zusammenhang ist die Trennung bestimmter Funktionen in den Städten wie Wohnen und Produzieren. Gleichzeitig soll die Umsiedelung von stark umweltverschmutzenden Betrieben in dünn besiedelte Gebiete gefördert werden, wobei ökologisch besonders wichtige Gebiete freilich ausgenommen sind (Art. 17 UmweltschutzG).

\section{(5) Verantwortungssystem}

Mit diesem System macht sich das chinesische Umweltrecht den stark kollektivistischen Charakter der chinesischen Gesellschaft zunutze: Die Regierung steht an der Spitze der Verantwortung für die Umwelt, sie setzt sich Umweltziele und gibt sie mit der damit verbundenen Verantwortung auf die ihr nachfolgenden Ebenen weiter, bis schließlich auch auf der untersten Stufe der Hierarchie eine konkret zu erfüllende Verantwortung festgeschrieben ist. ${ }^{50}$ Gleichzeitig werden einzelne Verwaltungsleiter, Betriebe oder sogar ganze Städte in eine spezielle Verantwortung genommen: In Verträgen mit der Zentralregierung oder den Umweltschutzbehörden werden konkrete Umweltziele vereinbart, die innerhalb einer bestimmten Zeit zu erfüllen sind. ${ }^{51}$ Je nach Typ des Vertrags folgen einer Erfüllung eine Belobigung oder eine Klassifizierung z.B. als "Schöne Stadt" ${ }^{52}$ oder aber handfeste wirtschaftliche Vorteile, wie etwa größere Freiheit im Management oder Gewinnbehalt-

47 48

49

50

51

52

State should use economic levers to stop polluters, in: China Daily, 13.6.1994, S. 4.

Vgl. Ning Minqing / Jiang Xia, A Potential Market for China's Environmental Protection Industry, in: China Environmental News, 2.4.1994.

Art. 12, 13, 17, 18, 22, 23.

Qu Geping, Environmental Management in China, Beijing 1991, S. 153.

Vgl. zum Zusammenspiel solcher Ziele und der Planung nach sozialistischem Muster z.B. $\mathrm{Li}$ Shidong, Environmental Protection Goals in Beijing's Overall Plans, in: China Environment Journal, 24.3.1994.

Vgl. zum "Wettbewerb um die 10 besten Städte in bezug auf Umweltschutz" z.B. Zhao Guanliang, Shanghai actively improves Urban Environment, in: China Environment Journal, 24.3.1994. 
rechte. Einer Nichterfüllung folgen Kritik oder die vertraglich vereinbarten Sanktionen, z.B. Ausbleiben einer Beförderung. Die Ergebnisse dieser Programme werden veröffentlicht. Außerdem hat China unter massiver Beteiligung der Öffentlichkeit ein Umweltschutzzeichen eingeführt. ${ }^{53}$

\section{(6) Standards, Emissionsgebühren, Lizenzen ${ }^{54}$, Steuern}

Hauptsächlich die National Environment Protection Agency hat in den letzten Jahren zahlreiche nationale Umweltstandards erlassen, die vor allem Wasser, Luft und Lärm betreffen. Wasser wird in fünf Kategorien eingeteilt und auf 35 verunreinigende Substanzen hin untersucht, die momentan noch in ihrer Konzentration in Abwässern gemessen werden; das System wird aber auf Massebasis umgestellt. Für Luft sind drei Kategorien festgesetzt worden, von denen die mittlere strenger, die unterste aber deutlich laxer als die entsprechenden Standards der WHO sind. ${ }^{55}$ Luftstandards werden auf Massebasis ermittelt, und ihre Strenge nimmt mit der Höhe des Schornsteins überproportional ab.

Jede diese Standards überschreitende Umweltverschmutzung ist ein Gesetzesverstoß. ${ }^{56} \mathrm{Er}$ ist verbunden mit der Pflicht, eine Emissionsgebühr zu bezahlen, deren Charakter zwischen Strafe und Kauf von Emissionsrechten liegt. ${ }^{57}$ Das Emissionsabgabesystem ist ein noncompliance-System. Die Abgabe wird nur fällig bei Übertretung des zulässigen Standards, nicht schon bei bloßer Emission innerhalb der zulässigen Grenzwerte. Eine wiederholte

Vgl. Sun Haidong, China Starts to Accept Products with EP Mark, in: China Environmental News, 12.7.1994; Liu Xiaojun, 6 Classes and 18 Kinds Products Acquired Environmental Mark, China Environmental News,, 23.3.1995.

Zu diesen drei Bereichen sehr ausführlich R. Nick Anderson u.a., Fn. 46, S. 29 ff.; Zhang Kunmin/ Jui Ruilin, Fn. 38, S. 245 ff.; Alan Krupnik / Sebastian Iona, Issues in Urban Air Pollution: Review of the Beijing Case, in: Environmental Working Paper No. 31 (World Bank), May 1990, S. 1, 9; Lester Ross / Mitchell Silk, PRC \& Taiwan, a Special Report, in: Environment Risk Yearbook Edition, September 1993, S. 62 ff.; Dirk Betke, Fn. 4, S. 72 f.

Vgl. R. Nick Anderson u.a., Fn. 46, S. 29 und Annex 3; Alan Krupnik/Sebastian Iona, Fn. 54, S. 1, 5 .

So ist Art. 28 UmweltschutzG zu verstehen: Zhang Kunmin / Jin Ruilin, Fn. 38, S. 125 f.

Diese Frage ist nicht ganz klar zu beantworten, es scheint sich aber die Überzeugung durchzusetzen, daß es sich nicht um eine Strafe, sondern eher um eine Art Steuer handelt, obwohl an der Illegalität der Emissionshandlung kein Zweifel besteht. Andererseits kann die Gebühr voll zu den Betriebskosten dazugerechtet werden; State should use economic levers to stop polluters, in: China Daily, 13.6.1994, S. 4. Siehe zum Thema z.B. R. Nick Anderson u.a., Fn. 46, S. 29 ff. und Annex 3 und 4; Bryan Bachner, People's Republic of China, in: Günther Handl, Yearbook of International Environmental Law, Volume 4, 1993, Oxford University Press, 1994; anderer Meinung im Hinblick auf Steuem allerdings Wang Jinnan / Yang Jintian / Lu Xinyuan / Zhu Dongqing, Untersuchung und Diskussion der Umweltsteuerpolitik Chinas in der Zeit des Wandels zur Marktwirtschaft (Shi chang jing ji zhuan xing qi zhong guo huan jing shui shou zheng ce de tan tao), in: Huanjing Kexue Jinzhan, April 1994, Nr. 2, S. 5 ff. 
Verletzung der Standards kann zu einer Strafe führen, die sich verdoppelt, wenn unrichtige Angaben über Emissionen gemacht werden. Die Höhe der Gebühren berechnet sich über einen Faktor, den die Provinzen festlegen, und steigt drei Jahre nach erstmaliger Gebührenerhebung jährlich um $5 \%$ an. Das bloße Zahlen der Gebühr entbindet den Verschmutzer nicht von seiner gesetzlichen Haftung im übrigen. Die Abgaben werden von den Finanzbehörden verwaltet und zu $20 \%$ dazu benutzt, die Umweltschutzbehörden zu finanzieren. Die restlichen $80 \%$ dürfen als Fonds nur dazu verwandt werden, Umweltschutzmaßnahmen zu finanzieren. Dabei können Betriebe, die selbst in den Fonds eingezahlt haben, nach Erschöpfen ihrer eigenen Mittel bis zu 80 \% der von ihnen eingezahlten Summe als Subvention oder Kredit für Umweltschutzmaßnahmen erhalten.

Darüber hinaus muß sich jeder Emittent bei den Umweltschutzbehörden registrieren lassen. In einigen Provinzen, Kreisen und Städten wird bereits mit Emissionslizenzen und Umweltabgaben experimentiert. Art. 15 des Gesetzes zur Prävention und Kontrolle von Wasserverschmutzung sieht sogar vor, daß auch für Abwässer, die die Standards nicht überschreiten, Abgaben gezahlt werden müssen; Art. 28 UmweltschutzG kennt aber nur die oben erwähnten Abgaben.

Steuern auf die Nutzung von Naturressourcen gibt es nur vereinzelt und weniger aus Umweltschutzgründen als vielmehr, um einen Konkurrenzausgleich zwischen verschiedenen Betrieben zu schaffen. Jedoch wurden 1984, 1985 und 1988 Steuervorteile für Nutzer von Abwässern, Abgasen und industriellen Rückständen gewährt, die aber z.T. wieder zurückgenommen worden sind. ${ }^{58}$ Im übrigen ist die Diskussion um Umweltsteuern weiter im Gange. 59

Neu ist ein gebührenpflichtiges Erlaubnissystem für die Benutzung von Waldflächen, das momentan noch schwer einzuschätzen ist. ${ }^{60}$

\section{(7) Fristsetzung}

Wenn ein Unternehmen dauerhaft Umweltstandards "in ernstem Ausmaß" verletzt, kann ihm die zuständige Regierung auf Vorschlag der Umweltschutzbehörde eine Frist zur Einhaltung der Standards setzen (Art. 29 UmweltschutzG). Wenn das Unternehmen die

Warum, ist nicht klar (Gründe werden nicht genannt in Wang Jinnan u.a., Fn. 57, S. 5 ff.).

Zuletzt $L u L i$, Umweltsteuern - Neue Maßnahmen für ein Regelsystem gegen öf fentliche Schäden (Huan jing shui - gui zhi gong hai de xin ju cuo), Faxue 3(1994, S. 36; vgl. auch National Environmental Protection Agency And State Planning Commission, Environmental Action Plan of China, 1991-2000, Beijing 1994, S. 42.

60 Xiong Zhihong, China Enforces Permit System for Forest Land Use, in: China Environmental News, 25.2.1995. 
gemachten Auflagen nicht innerhalb der gesetzten Frist erfüllt, kann ihm die Umweltschutzbehörde neben der Emissionsgebühr eine Strafe auferlegen, oder die zuständige Regierung kann den Betrieb suspendieren oder das Unternehmen schließen (Art. 39 UmweltschutzG). ${ }^{61}$

\section{(8) Untersuchung vor Ort}

Die Umweltschutzbehörden können zur Kontrolle der Gesetzeseinhaltung unangekündigte Inspektionen vor Ort durchführen (Art. 14 UmweltschutzG), wobei die inspizierten Unternehmen unter Strafandrohung zur Kooperation verpflichtet sind (Art. 35 UmweltschutzG). Wenn sich bei einer solchen Untersuchung herausstellt, daß ein Unternehmen vorgeschriebene Kontrollanlagen nicht installiert hat oder nicht benutzt, kann die Umweltschutzbehörde die Installation/Inbetriebnahme solcher Anlagen verlangen oder an Ort und Stelle die Produktion anhalten lassen (Art. $36 \mathrm{f}$. UmweltschutzG). Ähnliche Vorschriften finden sich in anderen Umweltschutzgesetzen. ${ }^{62}$

\section{(9) Haftung}

Das chinesische Umweltrecht sieht administrative, zivile und strafrechtliche Haftung vor.

(a) Bei der administrativen Haftung sind die Schadensersatzpflicht der Verwaltung gegenüber einem durch einen Fehler der Verwaltung Geschädigten ${ }^{63}$, Disziplinarmaßnahmen und administrative Strafen zu unterscheiden. Disziplinarmaßnahmen werden von Betrieben oder Behörden ihren eigenen untergeordneten Mitgliedern bei Fehlverhalten auferlegt, also z.B. Pflichtverletzung oder auch einer Umweltschädigung. ${ }^{64}$ Administrative Strafen können für jede die Umwelt schädigende Handlung, die nicht strafrechtsrelevant ist, von den Umweltschutzbehörden verhängt werden. ${ }^{65}$ Verschuldensabhängige Haftung darf nicht als Produktionskosten berechnet werden. ${ }^{66}$

61

62

63

64

65

66

Vgl. etwa Duan Songqiao, Two Beijing Enterprises Closed Down by Environmental Protection Bureau, in: China Environment Journal, 12.4.1994.

Z.B. Art. 18, 37 des Gesetzes zur Prävention und Kontrolle von Wasserverschmutzung, Art. 13 der Implementierungsregeln zum selben Gesetz.

Chen Hanguang / Piao Guang / Bian Zhu, Basis des Umweltgesetzes (Huan jing fa ji chu), Beijing 1994, S. $172 \mathrm{ff}$.

Betriebe sind meistens (zumindest indirekt) einem Ministerium untergeordnet. $\mathrm{Zu}$ dieser Art Maßnahme vgl. Art. 38, 45 UmweltschutzG, Art. 39 des Gesetzes zur Prävention und Kontrolle von Wasserverschmutzung.

Vgl. z.B. die z.T. empfindlichen Strafen für vier Unternehmen in Beijing: An Wei, Beijing Dealt With 4 Polluters, in: Beijing Daily, 30.3.1995.

Zhang Kunmin / Jin Ruilin, Fn. 38, S. 318. 
(b) Zivil haftet ${ }^{67}$ grundsätzlich, wer die Zivilgesetze verletzt und dadurch Schaden an Leib und Leben eines anderen oder an seinem Eigentum anrichtet. Im Umweltrecht ist aber die Ungesetzlichkeit der Handlung keine unbedingte Haftungsvoraussetzung: Eine Emission im Rahmen geltender Standards etwa kann Zivilhaftung begründen. ${ }^{68}$ Ebenso ist Verschulden bei der zivilen Umwelthaftung im Gegensatz zum allgemeinen Zivilrecht entbehrlich (Art. 106 ZivilG i.V.m. Art. 41 UmweltschutzG). Neben der zivilen können administrative und straf rechtliche Haftung kumulativ bestehen (Art. 120 ZivilG). Zwar braucht der Kläger nur die Evidenz des Kausalzusammenhanges nachzuweisen, eine Beweislast zugunsten des Geschädigten gibt es aber nicht. ${ }^{69}$ Schließlich gilt nicht die allgemeine Verjährungsdauer von zwei Jahren ab Schadensentstehung (Art. 135 ZivilG), sondern eine dreijährige, deren Berechnung erst beginnt, wenn der Geschädigte von dem Schaden erfahren hat oder erfahren konnte (Art. 42 UmweltschutzG i.V. m. Art. 141 ZivilG).

(c) Strafrechtlich werden nur Taten verfolgt, die zu schweren Schäden an geschützten Rechtsgütern geführt haben. Die Umweltschutzgesetze verweisen auf das Strafrecht, ohne i.d.R. selbst Straf vorschriften zu statuieren (z.B. Art. 43 f. UmweltschutzG). Das Strafgesetzbuch allerdings enthält kein eigenes Kapitel Umweltstrafrecht, sondern nur einzelne relevante Vorschriften in anderen Kapiteln. Die Revision mehrerer Umweltschutzgesetze bis 1997 soll differenziertere und angemessenere strafrechtliche Sanktionen erbringen. ${ }^{70}$

\section{Die Rolle der Bürger}

Was früher als Rolle der Massen beschrieben wurde und im Umweltschutzgesetz mit Überwachungsrechten (Art. 8), Recht und Pflicht, am Umweltschutz teilzunehmen (Art. 6) und Umwelterziehung (Art. 4) Niederschlag gefunden hat, diskutieren chinesische Rechtsgelehrte in letzter Zeit als Umwelt-Bürgerrechte ${ }^{71}$, ohne daß dabei momentan abzusehen ist, in welche Richtung sich diese neue Interpretation des Umweltrechts bewegt.

67

Dazu sollte 1995 eine rechtsvergleichende Dissertation an der Universität Trier von Zhu Guolin erscheinen.

68

Vgl. Factories lose pollution case to fish farm, in: China Daily, 11.1.1995, S. 2; dort mußten Fabriken umgerechnet $28.000 \$$ Schadensersatz an eine Fischfarm bezahlen, weil deren Fische durch die Abwässer der Fabriken verendet waren, obwohl die Abwässer sich im Rahmen der staatlichen Standards gehalten hatten.

Zhang Kunmin / Jin Ruilin, Fn. 38, S. 325 f.

70 Vgl. Shao Lihua, China to Promulgate More Environmental Protection Laws, in: China Environment Journal, 3.9.1994; Jiang Yue, China's Environmental Laws to Add Criminal Provisions, in: China Reform, 6.9.1994; Liu Wenren, Einleitende Untersuchung zu Umweltverbrechen in China (Wo guo huan jing fan zui chu bu yan jiu), in: Faxue Yanjiu 3/1994, S. 67.

Z.B. Chen Maoyun, Diskussion von Umweltbürgerrechten (Lun gong min huan jing quan), in: Zhengfa Luntan 1990, Nr. 6, S. 36 ff.; Hou Mingguang, Diskussion der (staats)bürgerlichen Umweltrechte (Lun gong min huan jing quan), in: Falu Kexue, 1991, Nr. 3, S. 42 ff. = Faxue 


\section{b. Aufbau der Umweltverwaltung}

An der Spitze der Umweltadministration steht die State Commission On Environmental Protection, ein dem Staatsrat (Regierung) ${ }^{72}$ unterstelltes Gremium, dem neben den Kommissionen für Wissenschaft und Technologie, für Wirtschaft und der für Planung die Ministerien für Stadt- und Landaufbau, für Forstwirtschaft, für Landwirtschaft, für Wasserressourcen, für Energie und eine Reihe industrieller Ministerien wie z.B. das für die chemische Industrie angehören. Dieses hochkarätig besetzte Gremium ist gemäß Art. 7 UmweltschutzG oberstes Organ für Umweltplanung und soll die Arbeit der gesamten Umweltadministration überwachen, planen, koordinieren und rechtliche wie politische Leitlinien formulieren.

Statt ein Ministerium für Umweltschutz einzurichten - eine Variante, die versuchsweise Mitte der 80er Jahre durchgeführt wurde, als dem Ministerium für Stadt- und Landaufbau diese Aufgabe zufiel -, kommen so alle in Umweltschädigung und -schutz verwickelte Ministerien und Staatskommissionen zusammen, um ihre Interessen zugunsten der Umwelt auf einander abzustimmen.

Allerdings tagt die Kommission nur wenige Male im Jahr. Faktisch höchste ständig arbeitende. Behörde ist daher die National Environmental Protection Agency (NEPA), die als Sekretariat der State Commission fungiert. Ihr unterstehen Umweltschutzbüros auf Provinz, Präfektur- und örtlicher Ebene sowie zahlreiche nationale und regionale Forschungsinstitute. Die NEPA ist zuständig für Umweltmanagement und -überwachung, für das Erstellen von Umweltqualitäts- (Art. 9 UmweltschutzG) und Emissionsstandards (Art. 10 UmweltschutzG). Der Behördenunterbau der NEPA, Umweltschutzbüros auf allen Ebenen, untersteht zugleich den jeweiligen Regierungen, die gemäß Art. 16 UmweltschutzG allein verantwortlich sind für die Umweltqualität in ihrem Zuständigkeitsbereich. ${ }^{73}$

Die eine Hauptaufgabe dieser Umweltschutzbüros liegt in der Registrierung von emittierenden Betrieben (Art. 27 UmweltschutzG), der Implementierung der drei simultanen Schritte (Art. 26 UmweltschutzG) und der Bewertung und Überwachung von EIA sowie Inspektionen vor Ort (Art. 13 ff. UmweltschutzG).

1992 D41, Nr. 1, S. 76 ff.; Li Yanfang, Etliche Fragen und Forschungen zu Umweltrechten (Huan jing quan ruo gan wen ti tan jiu), in: Falü Kexue 6/1994, S. 61; Wei Lianchun, Gesetzlicher Schutz von Umweltrechten (Huan jing quan de li fa bao hu), in: Faxue 6/1994, S. 38.

$\mathrm{Zu}$ den chinesischen Staatsorganen i.a. siehe Albert H.Y. Chen, Fn. 2, S. 39 ff. und 63 ff. Zum Ganzen vgl. z.B. Dirk Betke, Fn. 4, S. 73-75 mit Organigramm.

Aus dieser doppelten Bindung ergeben sich für die Umweltschutzbüros einige Probleme, die im folgenden Abschnitt darzustellen sein werden. 
Die andere Hauptaufgabe besteht darin, die Umwelt zu untersuchen, zu bewerten ${ }^{74}$ und Umweltschutzpläne zu erstellen, die die jeweils zuständige Regierung genehmigen muß (Art. 12 UmweltschutzG). Die NEPA muß nach Art. 11 UmweltschutzG ein Meßsystem etablieren, Normen dafür erstellen und ein Meßnetzwerk einrichten ${ }^{75}$ sowie wie alle Provinzumweltschutzbüros einmal jährlich über den Zustand der Umwelt berichten. ${ }^{76}$ Neben diesen Umweltschutzbehörden unter der State Commission gibt es fast in jedem der in ihr vertretenen Ministerien eigene Umweltadministrationen auf allen Ebenen. So verfügt z.B. das Ministerium für die chemische Industrie über ein eigenes Meßnetzwerk, eigene Standards und eigen Managementregularien. ${ }^{77}$ Außerdem haben verschiedene Forschungsinstitute $^{78}$ eigene Meßsysteme und -netzwerke aufgebaut. Theoretisch ist die NEPA für die Koordination all dieser Bereiche zuständig, hat aber keine Vormachtstellung.

Diese Organisationsstruktur ist wegen ihrer großen Reibungsverluste scharf kritisiert worden, was sogleich dargestellt werden soll.

\section{Probleme in der Rechtswirklichkeit}

Trotz der soeben vorgestellten umfangreichen Gesetzgebung und eines extensiven dezentralisierten Verwaltungsapparates (etwa 200.000 Menschen arbeiten in Chinas Umweltschutzadministration ${ }^{79}$ ) haben die letzten Jahre keine signifikante Verbesserung der Umweltlage in China erbracht. Es gehört zu den Widersprüchen des chinesischen Alltags, daß einerseits von Regierungsseite behauptet wird, die Implementierung des Umweltrechts

74

Vgl. dazu Yu Changhong, Report Sees Improved Ecological Environment in China, in: People's Daily, Overseas Edition, 4.6.1994; Li Wenqi, Environmental Pollution and Ecological Destruction Persist in China, in: Liberation Daily, 4.6.1994; Gesamtchinesischer Kommentar und Untersuchung von Quellen industrieller Umweltverschmutzung, Gesamtband (Quan guo gong ye wu ran yuan diao cha ping lun yu yan jiu zong lun), Beijing 1990 und Teilband (Fen lun) 1991; Chinas Umweltjahrbuch 1994 (Zhong guo huan jing nian jian), Beijing 1994.

In den Regelungen für das Management von Umweltmessungen ist im einzelnen ausgeführt, wie dies zu geschehen hat.

76 Vgl. dazu die Hinweise in Fn. 74 und Li Shi, 1994 Chinese Environmental Situation Bulletin Issued by NEPA, in: China Environmental News, 3.6.1996. Voller Text des NEPA-Umweltreports 1994 vgl. oben Fn. 4. Die Umweltreports erfüllen die Verpflichtung aus Art. 11 UmweltschutzG, nicht die aus Art. 12.

Ministry of Chemical Industry of the People's Republic of China, Environmental Protection of the Chemical Industry of China, Beijing 1992, S. 22.

78 Mehr und mehr versucht die chinesische Regierung, Spezialisten aus allen Branchen, möglichst auch aus dem Ausland, für kostenlose oder vom Ausland bezahlte Beratungstätigkeit zu gewinnen; vgl. Proceedings, The First \& Second Meetings of China Council for International Cooperation on Environment \& Development, Beijing 1994.

79

Etwas niedriger $R$. Nick Anderson u.a., Fn. 46, Annex 1, S. 6. 
funktioniere mit Raten bis zu $100 \%$ sogar in kleinen Unternehmen und das Umweltrechtssystem sei - von kleinen Schwächen abgesehen - komplett und funktionstüchtig ${ }^{80}$, und andererseits mit Chinas Agenda 21 die große Wende zu einem marktwirtschaftlichen Umweltschutzrecht eingeleitet werden soll, um den Unzulänglichkeiten des derzeit geltenden Systems zu entkommen. ${ }^{81}$

\section{a. "Technische Schwächen" der Rechtsgrundlagen}

Strukturell werden am chinesischen Umweltrecht vor allem die Inkompatibilität des in der Planwirtschaftsphase entstandenen Rechts mit der jetzigen marktwirtschaftlichen Struk$\operatorname{tur}^{82}$, die Unstimmigkeit von alten und neuen, örtlichen und nationalen, spezifischen und

allgemeinen und Regelungen des Umweltrechts und anderer Rechtsgebiete ${ }^{83}$, die Betonung auf end-of-pipe-Kontrolle statt Förderung sauberer Produktion ${ }^{84}$ und die enormen Reibungsverluste zwischen den einzelnen Teilen der Umweltschutzverwaltung und zwischen den Umweltschutzbehörden und den jeweiligen Regierungen bzw. anderen Abteilungen (z.B. in Fachministerien) ${ }^{85}$ gerügt. Ferner werden Lücken im Gesetzesschutz aufgezeigt in den Bereichen Ver-Wüstung von Gebieten, Müll, Sonder- und Giftmüll, Bodenschutz, Verwertung von Abfällen und Ressourcen, Schutz natürlicher Ressourcen und Bürgerrechte

80

So z.B. State Planning Commission, People's Republic of China, Comprehensive Utilization of Chinese Wastes, Beijing, ohne Datum; Ministry of Forestry of the People's Republic of China, The Protection of Wild Fauna and Flora in China, Beijing 1992; Yang Jilin für die China NEPA, Environmental Protection of Township Industries in China, Beijing 1992; Ministry of Chemical Industry of the People's Republic of China, Fn. 77. Neuerdings wird diesen Zahlen allerdings ebenfalls von of fizieller Seite entgegengetreten, so für die EIA Gao Jie, Is environmental impact assessment a shield?, in: China Environmental News,, Dez. 1994, S. 4 f.

China's Agenda 21, Fn. 7; Priority Programme für China's Agenda 21, Fn. 21, S. 1 ff.; Future needs growth, environment, in: China Daily, 8.7.1994, S. 1; Green plan receives overseas support, in: China Daily, 24.6.1994, S. 1.

82 Priority Programme for China's Agenda 21, Fn. 21, 1-1 A-1; Ma Guancong, Entwicklung der Marktwirtschaft und Vervollkommnung des Umweltrechtssystems ( $F$ a zhan shi chang jing ji yu wan shan huan jing fa zhi), in: Faxue Yanjiu 5/1994, S. 52, 54 sub 4 (si); Wei Lianchu, Über die Vervollkommnung des Umweltrechtssystems im marktwirtschaftlichen System (Lun wan shan shi chang jing ji ti zhi xia de huan jing fa lu ti xi), in: Faxue Pinlun 5/1994, S. 80.

83 Feng Jianmei, Die Marktwirtschaft und die Vervollkommnung der Umweltgesetzgebung (Shi chang jing ji yu huan jing li fa de wan shan), in: Zhongguo Huanjing Guanli, 1994, Nr. 2, S. 34 f.

Priority Programme for China's Agenda 21, Fn. 21, 3-1-1.

85 Was sich z.B. in der Inkompatibilität der von den verschiedenen Meßnetzen gemessenen Daten zeigt. Robert D. Perlack / Milton Russell, Energy and Environmental Policy in China, in: Annual Revision of Energy and the Environment, Vol. 16, 1991, S. 205, 223; R. Nick Anderson u.a., Fn. 46, Annex 3, S. 16 ff.; Feng Jianmei, Fn. 83, S. 34 f. 
im Umweltbereich ${ }^{86}$. Schwer wiegt die Kritik, das gesamte Umweltrechtssystem sei ausgehend von Partikularinteressen und ohne integrierende Planung geschaffen worden. ${ }^{87}$

Außerdem werden zahlreiche Einzelpunkte der gesetzlichen Regelungen angegriffen, etwa $\mathrm{da} ß$ die Emissionsgebühren generell zu niedrig seien, um annähernd die notwendigen Kosten für Präventionseinrichtungen zu erreichen, daß keine Anreize geschaffen würden, die Standards nicht nur einzuhalten, sondern sogar zu unterschreiten, daß Gebühren als Produktionskosten abgerechnet werden können. ${ }^{88}$ Am Haftungssystem wird z.B. moniert, daß es keinen Schadensersatz für Ersatz nichtmaterieller und nur Ersatz bestimmter körperlicher Schäden kennt. ${ }^{89}$

Diese Kritik ist sicher berechtigt, die von ihr angeprangerten Unzulänglichkeiten erklären aber noch nicht, warum die Umwelt trotz der rechtlichen und administrativen Anstrengungen in einem so schlechten Zustand wie dem gegenwärtigen ist. Nach der Untersuchung der Durchsetzung wird daher nach einem weiteren grundsätzlichen Problem zu suchen sein (s.u. IV).

\section{b. Durchsetzung von Umweltrecht}

Nicht selten passiert es, daß Anordnungen der Umweltschutzbüros nicht befolgt werden. So ist z.B. der Fall einer pharmazeutischen Fabrik bekannt, die trotz mehrfacher Abmahnung durch die Umweltschutzbehörden seit 20 Jahren (!) giftige Dämpfe emittiert und für die Erkrankung zahlreicher Anwohner verantwortlich ist. Erst jetzt wurde ihr die Produktion bestimmter Stoffe von der zuständigen Regierung verboten. Dieses Verbot hält sie ein. ${ }^{90}$

Oft können sich die Umweltschutzbehörden einfach nicht gegen Betriebe durchsetzen, da diese unter dem Schutz der lokalen Regierung stehen. Im gerade beschriebenen Fall war die Fabrik einer der wenigen Devisenverdiener der Stadt Wuhan. Generell tut sich der Staat schwer, weil er in den meisten Fällen auf beiden Seiten steht: Er ist Verschmutzer und Kontrolleur zugleich. ${ }^{91}$

China's Agenda 21, Fn. 7; Priority Programme for China's Agenda 21, Fn. 21, 1-1; Feng Jianmei, Einige Gedanken zur Vervollkommnung der Umweltrechtsgesetzgebung meines Landes (Wan shan wo guo huan jing li fa de ji dian jian yi), in: Faxue yu Shehui, 1993, Nr. 5, S. 19 f.

Feng Jianmei, Fn. 83, S. 34 f.

R. Nick Anderson u.a., Fn. 46, S. 28 ff. und Annex 1 und 2.

Feng Jianmei, Fn. 86, S. 19 f.; eine geistige Behinderung wird nicht als in Geld ausdrückbarer Schaden anerkannt: Zhang Kunmin / Jin Ruilin, Fn. 38, S. 338. 
Der mangelnden Rechtstradition Chinas und einer bemerkenswerten Rechtsunkenntnis aller Beteiligten ist es wohl zuzuschreiben, daß Gebühren, Strafen und Anordnungen fast immer Gegenstand von Verhandlungen sind und nur sehr selten autoritativ festgesetzt und eingehalten werden. ${ }^{92}$ Persönliche Beziehungen und die faktischen Machtverhältnisse vor Ort sind häufig wesentlich wichtiger als geschriebenes Recht. ${ }^{93}$

Auch bei den Umweltschutzbehörden selbst ist nach Ursachen für die mangelnde Durchsetzung des Rechts zu suchen: Vor allem lokale Umweltschutzbüros sind häufig materiell schlecht ausgestattet, und die Mitglieder haben keine oder nur eine unzureichende Ausbildung. Es fehlt an einer Supervisionsinstanz für die Kontrolleure, so daß Veruntreuung und Zweckentfremdung von Geldern nicht selten vorkommen. ${ }^{94}$ Häufig fehlt den Umweltschutzbüros die Motivation, den Teil der Emissionsgebühren einzutreiben, der in den Umweltschutzfonds fließt, und sie geben sich mit den $20 \%$ zufrieden, die ihrer eigenen Finanzierung dienen. ${ }^{95}$ Außerdem häufen sich die Berichte, denen zufolge die Umweltschutzbehörden über wenig Autorität bei der Bevölkerung verfügen und sich offenen Anfeindungen ausgesetzt sehen. ${ }^{96}$

So gesehen liegt in der - an und für sich höchst sinnvollen - Dezentralisierung der Umweltschutzverwaltung ein Hauptproblem mangelnder Durchsetzung von Umweltrecht. ${ }^{97}$ Der NEPA selbst sind alle diese Probleme hinreichend bekannt. Sie versucht, sie durch Pläne für die Umweltbüros und häufige Treffen und Austausch mit den Regierungen auf allen Ebenen in den Griff zu bekommen. ${ }^{98}$ Der nötige bahnbrechende Erfolg steht jedoch weiterhin aus. ${ }^{99}$

Priority Programme for China's Agenda 21, Fn. 21, 1-1 B-1; Robert D. Perlack / Milton Russell, Fn. 85, S. 205, 226; Qu Geping, Fn. 50, S. 318.

93 Vgl. nur Zhang Jinsheng, Wild Animals Smuggling Rampant Along National Highway in China, in: Legal Daily, 17.8.1994; er berichtet über Fälle von Schmuggel geschützter Tiere im großen Stil, der aufgedeckt wurde, ohne da $B$ die Verantwortlichen bestraft wurden: Es waren Polizei- und Armeeoffiziere mit guten Beziehungen.

94 Qu Geping, Fn. 50, S. 318; Chen Qian, Serious Default and Appropriation of Sewage Discharge Tax in China, in: China Environmental News, 7.5.1994. Qu Geping, Fn. 50, S. 318.

Siehe etwa Xiong Zhihong, Forest police beaten on Wuyi Mountains, in: China Environmental News, Dezember 1994, S. 2.

97 So auch Bryan Bachner, Coming Home to Roost: Pollution, Law and Economics in the People's Republic of China, in: Georgetown International Environmental Law Review, Vol. V: Issue 3, Summer 1993, S. 635, 646; Robert D. Perlack/Milton Russell, Fn. 85, S. 205, 226. Interview mit Frau Han Min LL.M., Regulation Division of Policy and Regulation Department der NEPA, am 14.3.1995.

Vgl. z.B. Zhu Da, Critical Situation of Law-abiding and Law-executing in China's Industrial Environment, in: China Environmental News, 24.11.1994. 
Auf seiten der (meist staatlichen) Betriebe fehlt es an der Durchschlagskraft finanzieller Argumente, so lange Energie zu einem hoch subventionierten Preis geliefert wird, Strafen und Gebühren notfalls an den Abnehmer weitergegeben werden (was mangels echter Konkurrenz kaum schadet) und die Betriebe weder selbst über ihr Wirtschaften entscheiden können, noch für Kosten und Verluste zur Verantwortung gezogen werden. ${ }^{100}$

Von den Justizorganen schließlich ist ebenfalls keine Hilfe in dieser Hinsicht zu erwarten, denn genau wie die Umweltschutzbehörden leiden sie an schlechter Ausbildung und sind nicht im mindesten unabhängig von den lokalen Regierungen und ihren Interessen. Im Zweifelsfall können höhere Regierungsvertreter oder Parteikader kraft ihrer Autorität Gerichtsurteile nach Belieben aufheben oder Gerichte anweisen, Fälle neu zu verhandeln und zu bestimmten Ergebnissen zu kommen. Das stärkt weder das Selbstvertrauen der Gerichte und Staatsanwaltschaften, noch dient es dazu, ihnen bei den Betrieben und Bürgern ein Ansehen als unabhängige, überparteiliche und vor allem letztlich entscheidende Instanz zu verschaffen. ${ }^{101}$

\section{c. Umweltrechtsfälle}

Im kaiserlichen China galt es als unanständig und verwerflich, vor Gericht die Lösung von Konflikten zu suchen. So erklärt sich, daß bis heute nur sehr wenige Streitigkeiten tatsächlich gerichtlich entschieden werden, meist einigen sich die Parteien mehr oder weniger gütlich in Schlichtungsinstanzen.

Gleichwohl sieht das Umweltrecht zahlreiche Haftungsarten vor, die letztlich von Gerichten zu überprüfen sind, und ermutigt die Bürger, Gesetzesverstöße anzuzeigen oder selbst als Kläger aufzutreten (z.B. Art. 6, 41 UmweltschutzG). In letzter Zeit scheint sich tatsächlich ein Wandel im Verhalten der Bürger anzuzeigen, und es ist von einer zunehmenden Bereitschaft die Rede, vor Gericht zu ziehen. ${ }^{102} \mathrm{Da}$ auch nach gerichtlichen Urteilen, soweit sie überhaupt bekannt werden, häufig viele Fragen offen bleiben, verdeutlicht das folgende Beispiel:

R. Nick Anderson u.a., Fn. 46, S. 30 ff.; China's Agenda 21, Fn. 7, S. 9; Wang Mingyun, Erste Sondierungen des Umweltrechtssystems unter < dem Einfluß> der Marktwirtschaft (Shi chang jing 101 ji xia de huan jing fa zhi chu tan), in: Zhongguo Huanjing Guanli, 1994, Nr. 2, S. 4 ff.

Siehe dazu den 5. Teil (S. 243 ff.) in Lester Ross / Mitchell Silk, Fn. 42; Chinas Juristen im Aufbruch? Der Aufstieg der Rechtsanwälte und die Defizite des Gerichtswesens, in: China aktuell, Juli 1995, S. 555

102 More Chinese follow Qiuju's example by going to the courts, in: China Daily, 14.7.1994, S. 4. 
Es wird berichtet, daß eine Baumaterialienfabrik in der Provinz Jilin seit 1990 umliegende Felder verseucht hatte, weil sie trotz gegenteiliger Anweisungen des zuständigen Umweltschutzbüros Flugasche offen lagerte, die verwehte. Die Frucht- und Gemüseerträge von 294 Landwirten der Umgebung fielen um $10 \%$, was einem Verlust von ca. 700.000 RMB entsprach. Die Landwirte zogen gemeinsam gegen die Fabrik vor Gericht, das ihnen Recht gab und die Fabrik zum Ersatz des Schadens in voller Höhe verurteilte. ${ }^{103}$

Es fallen folgende Punkte auf: Erstens, daß 294 offenbar dörfliche oder kleinstädtische Kläger gemeinsam vor Gericht gezogen sein sollen, ist in hohem Grade unwahrscheinlich und spricht eher dafür, daß sie von politischer Seite Unterstützung bekommen haben, womit a priori ein fairer Prozeß zweifelhaft erscheint. Zweitens konnte offenbar die Fabrik jahrelang Weisungen des örtlichen Umweltschutzbüros ungestraft ignorieren. Es bedurfte also mehr als der bloßen Tätigkeit der Umweltschutzbehörden, um irgendeine Haftung zu erwirken. Drittens: Außer der zivilen Haftung scheint die Fabrik keine weiteren Sanktionen hinnehmen zu müssen. In einem solchen Fall würde man zusätzlich administrative (bestrafende und disziplinarische) erwarten. Viertens, daß die Bauern einen zehnprozentigen (!) Ernterückgang über Jahre und außerdem den Kausalzusammenhang mit der Flugasche nachgewiesen haben sollen, ist sehr unglaubwürdig ${ }^{104}$, so daß auch hier der Verdacht erhärtet wird, es habe sich um einen Schauprozeß gehandelt. ${ }^{105}$

Die Anzahl der bekannten Umweltprozesse ist insgesamt gering, und es scheint nur festzustehen, daß zumindest Firmen mit ausländischer Finanzbeteiligung vor Gericht, wenn überhaupt, mit einem fairen Prozeß, so wenigstens nicht mit Nachsicht rechnen dürfen ${ }^{106}{ }^{107}$

103

Farmers reap $\$ 80,000$ in damages, in: China Daily, 21.7.1994, S. 3.

Laut Angaben in China tätiger ausländischer Rechtsanwälte und Geschäftsleute ist Buchführung sogar in vielen großen Staatsbetrieben unbekannt, und so erscheint es extrem unwahrscheinlich, daß Kleinbauern einen genauen Überblick über ihre Produktion haben.

105 Außerdem fallen zwei weitere Punkte auf: Die Fabrik hätte die Flugaschenlagerung in ihrem EIA angeben und die drei simultanen Schritte einhalten müssen. Mit offen lagernder Flugasche hätte die Inbetriebnahme nicht genehmigt werden dürfen. Die Rolle des Umweltschutzbüros bleibt dubios. Außerdem bekommt der Fall dadurch eine pikante Note, daß es sich bei der Fabrik um ein Abfall nutzendes Unternehmen handelt, sie also zur Umweltschutzindustrie zählt. Inwieweit dies 106 hier eine Rolle spielte oder hätte spielen können, ist unklar.

Das lehrt der erste bekanntgewordene Umweltrechtsfall "Kaida", s. Zhang Kunmin / Jin Ruilin, Fn. 38, S. 287 f.; Bryan Bachner, Fn. 25, S. 229, 244; sehr ausführlich auch Joseph R. Profaizer, Fn. 18, S. 320, 337-341.

107

Weitere Fälle in: Bevölkerung mit Umweltbedingungen unzufrieden, in: China aktuell, Juli 1992, S. 451; Three importers of chemical waste face court action, in: China Daily, 22.6.1994, S. 3; Factories lose pollution case to fish farm, in: China Daily, 11.1.1995, S. 2; Hu Yueping, Guizhou Improves Controls on Heavy-Polluting Enterprises, in: People's Daily, 14.9.1994. Nicht zu Gerichtsverhandlungen kam es trotz z.T. erheblicher rechtlicher Zweifel am Vorgehen der Behörden bei: Nanjing Municipal Waterworks Construction Company Case, in: China Law and 


\section{Umweltpolitik}

\section{Rolle von Ökonomie und innerer Sicherheit}

Umweltschutz in China soll zwar mit der Wirtschaft eine dialektische Einheit bilden, hat aber vorerst hinter dieser zurückzustehen: Fast im selben Atemzug mit der Postulierung des sustainable development schreibt Chinas Agenda 21 fest, Voraussetzung dafür seien erst einmal wirtschaftliches Wachstum und nationale Stärke, und zwar notfalls auf Kosten der Umwelt. ${ }^{108}$ Mit nationaler Stärke ist dabei nicht nur die nach außen, sondern auch die des Staates gegen seine eigenen Bürger gemeint: Unabhängige Umweltorganisationen gibt es in China mit wenigen Ausnahmen ${ }^{109}$ allenfalls auf lokaler Ebene ${ }^{110}$, "private" Forschungseinrichtungen oder Verbände ${ }^{111}$ stehen unter strenger Kontrolle, Umweltopposition wird nicht geduldet. $^{112,113}$

Practice, 24.9.1990, S. 39 f.; NEPA cracks down on waste shipment, in: China Daily, 14.6.1994, S. 3.

108

China's Agenda 21, Fn. 7, S. 4. Im selben Sinn Zhou Dadi, Fn. 7, S. 221, 228.

109 Die "Friends of Nature" (FoN) sind eine unselbständige Umweltorganisation, die der nichtstaatlichen Kulturorganisation angegliedert ist (es darf nach chinesischem Gesetz nur je eine nichtstaatliche Organisation in einem Bereich geben; der Umweltbereich soll offenbar vorerst gar nicht besetzt werden, deshalb wurde der Ausweg der angegliederten Organisation gewählt). Interview mit Liang Cong Jie, Präsident der FoN, am 17.3.1995. Eine weitere, von der Dissidentin Dai Qing gegründete Gruppe ist nach einjährigem Bestehen als Beijing Environmental Translation Centre wegen der politischen Probleme gezwungenermaßen nur nach außen tätig, versteht es aber, sich mit geschickter Argumentation und Vorgehensweise (es werden nur Artikel staatlicher Zeitungen übersetzt und ins Ausland verschickt) im Bereich staatlicher Duldung zu halten. Eine Betätigung in China sei erst nach einer politischen Liberalisierung und Entpolitisierung des Umweltschutzes vorstellbar. Interview mit der Gruppe am 14.3.1995 in Beijing. Jüngst wurde bekannt, daß es eine weitere private Gruppe gibt, die mit modernster Computertechnologie Informationen über die Umwelttechnologie in China verbreiten will (Institut für Umwelt und Entwicklung), vgl. Sheila Tefft, Chinesische Pionierin im Cyberspace, in: die tageszeitung, 9.8.1995, S. 9.

Interview am 8.3.1995 mit Herm Zhao Xing (Direktor der Internationalen Abteilung) und Frau Ding Jing (Internationale Abteilung, Herausgeberin) der China Environmental News.

111 Die 1993 gegründeten "Friends of Nature" sind die einzige Umweltorganisation in China überhaupt, die Bürger aufnehmen darf, vgl. Green to be seen and heard with watchdog, in: China Daily, 23.6.1994, S. 3. Sie steht jedoch unter dem ständigen Druck, aus politischen Gründen nicht zu viele Mitglieder zu bekommen (derzeit hat sie etwa 100). Thr politischer Aktionsradius ist extrem eingegrenzt und weit von dem entfernt, was nötig wäre, um Umweltbewußtsein in der Bevölkerung fördern oder gar die Regierung effektiv kontrollieren zu können. Interview mit Liang Cong Jie, Präsident der FoN, am 17.3.1995.

112

Dies wird vor allem im Zusammenhang mit AKW und dem Drei-Schluchten-Projekt deutlich: Bryan Bachner, Fn. 25, S. 229, 248, aber auch mit dem Verbot von Büchern zur Situation der Umwelt in China nach dem Massaker vom 4.6.1989.

113 Zur (möglichen) Rolle der Konsumenten im Zusammenhang mit Umweltschutz anhand der Sonderwirtschaftszonen s. Joseph R. Profaizer, Fn. 18, S. 320, 346 f. 
Das Umweltrecht will zwar die Bürger als Überwacher von illegalen Handlungen gegen die Umwelt, das Entscheidungsmonopol der Regierung aber soll nicht infragegestellt werden. ${ }^{114}, 115$ Den Widerspruch, der darin liegt, daß eine schlecht informierte (mangels freier Presse $^{116}$ und Umweltbewegung) Bevölkerung nur schwer das für eine Partizipation am Umweltschutz nötige Umweltbewußtsein wird entwickeln können, sieht die chinesische Regierung nicht - oder sie nimmt ihn hin. ${ }^{17}$ Begreift man die derzeitige Diskussion in der chinesischen Fachliteratur um Umweltbürgerrechte von dieser Seite her, versprechen vor allem solche Standpunkte zukünftige Veränderungen in diesem Bereich, die nicht von vornherein Umweltrechte nur im Zusammenhang mit Pflichten gewährt sehen wollen. ${ }^{118}$

114 Carlos Wing-Hung Lo, Environmental Management By Law in China: The Guangzhou Experience, University of Hong Kong, Centre of Urban Planning and Environmental Management, 1993, S. 3 f. Siehe auch jüngst Agnes Cheung, Environment fear "may spark mass exodus", in: South China Morning Post, 1.3.1995.

115 Die Bedeutung der Umweltbewegung für den Umweltschutz und seine gesellschaftliche Akzeptanz etwa in Deutschland sind allerdings bei der NEPA durchaus bekannt und werden unter diesem Aspekt ausdrücklich gutgeheißen. Die politische Lage in China sei allerdings derzeit (noch) nicht reif für eine solche gesellschaftliche Umweltbewegung nichtstaatlicher Art. Interview mit Frau Han Min LL.M., Regulation Division of Policy and Regulation Department der NEPA am 14.3.1995. Dennoch war es NEPA, die eine wichtige Rolle bei der Zulassung der Friends of Nature gespielt hat (wenn auch nur indirekt: Eine Zulassung als selbständige nichtstaatliche Organisation hat sie verwehrt, gleichzeitig aber den jetzt eingeschlagenen Weg angeregt, sich vorerst als Unterorganisation einer anderen nichtstaatlichen Organisation zu formieren). Interview mit Liang Cong Jie, Präsident der FoN, am 17.3.1995. Dies ist nicht anders zu erklären als damit, daß die NEPA die Existenz dieser nichtstaatlichen Organisation im Rahmen der politisch Machbaren fördern will.

116 Auch die häufig kritische Berichterstattung in der chinesischsprachigen Presse darf nicht darüber hinwegtäuschen, daß Zensur stattfindet: Es wird von seiten der Regierung sehr genau darauf geachtet, daß die Kritik "konstruktiv" ist, also sich vor allem nicht gegen die Zentralregierung wendet. Im Gegenteil setzt die Regierung die Presse gezielt dafür ein, unliebsame Entwicklungen auf Provinzebene oder darunter zu brandmarken. Information der deutschen Botschaft Peking und Interview am 8.3.1995 mit Herm Zhao Xing (Direktor der Internationalen Abteilung) und Frau Ding Jing (Internationale Abteilung), Herausgeberin der China Environmental News.

Nur vor diesem Hintergrund können die Umwelt-Erziehungskampagnen der 80er Jahre richtig eingeordnet werden: Sie blieben fast völlig wirkungslos, obwohl sie mehrere hundert Millionen Menschen erreichten. Entsprechend ist Skepsis angezeigt, wenn die Regierung nun wieder Anstrengungen ankündigt, das Umweltbewußtsein der Bevölkerung durch Erziehung zu steigern. Vgl. Umweltbewußtsein und Umwelterziehung, in: China aktuell, Juni 1995, S. 469.

Feng Jianmei, Fn. 83, S. 34 f. 


\section{Zukunftspläne für die Bewältigung der Umweltprobleme}

In fernerer Zukunft allerdings, so verspricht zumindest die Regierung in Chinas Agenda $21^{119}$, soll "sustainable development" nicht mehr zulasten der Umwelt gehen, sondern zugunsten von Wirtschaft, Gesellschaft und Umwelt. Offenbar ist die Regierung willens, die Agenda in mittel- und langfristige Entwicklungspläne aufzunehmen und hat bereits eine Prioritätenliste für bestimmte Projekte erarbeitet. ${ }^{120}$ Dazu gehört auch eine stärkere Beteiligung der Bürger an der Gesetzgebung, zumindest in Form einer besseren Rückkoppelung.

Kurzfristig jedoch hat die NEPA angekündigt, das bestehende System zu verbessern: Bis Ende 1996 sollen die wesentlichen Lücken in der Gesetzgebung geschlossen sein und Verschmutzer mit viel höheren Abgaben, Emissionsabgaben bereits unterhalb des Standards und harten Strafen (Schließung von Unternehmen und Freiheitsentzug für Hauptverantwortliche) bei Nichtbefolgen von Gesetzen und behördlichen Anweisungen zu umweltfreundlichem Verhalten animiert werden. ${ }^{122}$

\section{3. "Marktwirtschaftliche" Lösungen für Umweltprobleme}

Parallel zur Umwandlung Chinas von einer sozialistischen Plan- in eine Marktwirtschaft sozialistischer Prägung haben sich auch Bemühungen entsponnen, im Umweltrecht marktwirtschaftliche Mechanismen zu entdecken oder, wo diese fehlen, sie für das Umweltrecht zu fordern. Die Idealvorstellung für die chinesische Regierung ist, daß sie sich auf makroskopische Regelungen beschränkt und im übrigen den Markt die Verteilung und umweltfreundliche Nutzung von Natur und Ressourcen regeln läßt. ${ }^{123}$

Verf rüht scheint allerdings das Urteil einiger ausländischer Beobachter, in China habe sich ein dezentraler Marktzugang als Lösung der Umweltprobleme etabliert (und dies sei auch adäquat): China ist immer noch weit davon entfernt, eine reine Marktwirtschaft zu sein ${ }^{124}$, und daher sind marktwirtschaftliche Instrumente zur Verhinderung und Kontrolle von

119

China's Agenda 21, Fn. 7, S. 4; vgl. auch National Environmental Protection Agency And State Planning Commission, Fn. 59, v.a. S. $1 \mathrm{ff} .42 \mathrm{ff} ., 71 \mathrm{ff}$.

120

Priority Programme for China's Agenda 21, Fn. 21.

121 China's Agenda 21, Fn. 7, S. 4 f.

122 Zhu Baoxia, Polluters to be fined or jailed, in: China Daily, 18.7.1994, S. 1.

123

124

China's Agenda 21, Fn. 7, S. 1 ff.

R. Nick Anderson u.a., Fn. 46, Annex 4. 
Umweltschutz nicht bereits an und für sich sinnvoll ${ }^{125}$, sondern es ist in jedem Fall zu prüfen, ob sie, gemessen an der jeweiligen Rechts- und Wirtschaftssituation Chinas, vernünftigen Nutzen bei vertretbaren Kosten erbringen. Experimente mit "extremen" marktwirtschaftlichen Instrumenten, die sogar in westlichen Industrienationen mit fortgeschrittenem Umweltrecht und "reiner" Marktwirtschaft umstritten sind ${ }^{126}$, sind im Zusammenhang mit China Gegenstand einer wissenschaftlichen (und teilweise stark ideologischen) Auseinandersetzung. ${ }^{127}$ Es ist gewiß richtig, im Interesse einer kontrollierten Verbesserung von Umweltrecht und Umweltsituation in China auf extreme und in ihren Auswirkungen wenig gesicherte Maßnahmen vorerst zu verzichten. ${ }^{128}$

Die unstreitig vorhandenen marktwirtschaftlich wirkenden Anreizsysteme (z.B. Emissionsabgaben, Steuererleichterungen, Förderung der Eigenverantwortlichkeit der Betriebe für ihr Wirtschaften) sind, richtig durchgesetzt, eher erfolgversprechend. Im übrigen spricht der weltweite Rückzug der sozialistischen Planwirtschaft an sich noch nicht dafür, daß die "planwirtschaftlichen" Ansätze im chinesischen Umweltrecht (Umwelt-, Raum-, Wirtschaftspläne) unbrauchbar sind. ${ }^{129}$ In der heutigen Situation scheint eine Mischung aus gemäßigten marktwirtschaftlichen Ansätzen mit relativ stark ausgeprägten bürokratischautoritativen (Fristsetzung, EIA, drei simultane Schritte, Haftungssystem etc.) und spezifisch chinesischen (Verantwortungssystem) Lösungen am ehesten adäquat. ${ }^{130}$ Bei der NEPA selbst, wo die zukünftige Entwicklung des Umweltrechts wesentlich mit vorbereitet wird, ist die Diskussion, welcher Ansätze man sich vorrangig bedienen sollte, of fenbar in vollem Gang, und es ist nicht abzusehen, zu welchem Ergebnis sie führen wird. ${ }^{131}$

Inwieweit in Zukunft eine Anpassung des Umweltrechts an ein mehr marktwirtschaftliches Wirtschafts- und ein verändertes Gesellschaftssystem (vor allem, wenn dies mehr von Bürgerrechten geprägt ist) sinnvoll ist, wird nicht zuletzt auch davon abhängen, inwieweit sich die Rahmenbedingungen zugunsten eines Rechtsstaats mit berechenbarer, unabhängiger

125 126

Carlos Wing-Hung Lo, Fn. 114, S. 3.

(Ver)käufliche Emissionsrechte etwa oder sogar die völlige Überlassung von Preisen für Umweltfaktoren an den freien Markt.

127 Nachweise bei Bryan Bachner, Fn. 25, S. 229, 230 ff.; vgl. auch die zurückhaltende Stellungnahme in R. Nick Anderson u.a., Fn. 46, Annex 4.

128 Bryan Bachner, No Law, No Sky: Economic Development and Environment in the Fifth Dragon, inJ. Cheng / S. MacPherson (eds.), Development in Southern China, Hong Kong 1995.

129 So auch Bryan Bachner, Fn. 97, S. 635, 639 f.

130 Das heißt freilich nicht, daß das existierende chinesische Recht auch inhaltlich im einzelnen in sich konsistent ist, nur weil es eine solche Mischung der Kategorien aufweist: Denn neben der Durchsetzung fehlt es vielmehr an einem klaren inhaltlichen Konzept innerhalb der und über die Kategorien hinweg, dazu sogleich unter 4.

131

Interview mit Frau Han Min, LL.M., Regulation Division of Policy and Regulation Department der NEPA am 14.3.1995. 
Justiz, rule of law und einer verantwortungsvollen und freiwillig teilhabenden Öffentlichkeit verändern.

\section{Eine klare Linie in Chinas Umweltrecht und -politik?}

Westliche Beobachter attestieren China erstaunlich häufig, es verfüge über ein in sich konsistentes Umweltrechtssystem, dessen Problem lediglich in der Durchsetzung liege. ${ }^{132}$ Die Chinesen selbst sehen das nur teilweise so und verlangen, wie bereits erwähnt, recht einhellig Anpassung des Umweltrechts an die Marktwirtschaft.

Insgesamt muß man sich wohl dem Urteil Bachners anschließen, daß hinter dem bestehenden Unweltrechtssystem offenbar keine einheitliche Idee steht ${ }^{133}$ und daß Umweltbelange im Zweifel hinter Wirtschaftswachstum und innerer Sicherheit zurückzustehen haben, weil die chinesische Regierung allem Anschein nach bisher nicht gewillt war, in einem klaren, in sich konsistenten Umweltrechtssystem mit reellen Rahmenbedingungen für seine Durchsetzung ein Bekenntnis für den Umweltschutz abzulegen, das ihn gegenüber wirtschaftlichem Wachstum und innerer Sicherheit zumindest gleich wichtig macht. ${ }^{134}$

Darauf deuten jedenfalls die zahlreichen Unschärfen in den Gesetzen, die Verwässerung der administrativen Komponenten und Berücksichtigung aller erdenklichen Partikularinteressen bis zu direkten Widersprüchen in den Gesetzen hin ${ }^{135}$ sowie die mehr oder weniger unverblümten Äußerungen hochrangiger Offizieller, daß ein westliches Interesse an chinesischem Umweltschutz nur gegen entsprechende technologische und finanzielle Hilfsleistungen realisierbar sei. ${ }^{136}$

Und das ist schließlich wohl auch der Grund dafür, daß trotz des chinesischen Umweltrechtssystems die Umweltsituation im heutigen China so verheerende Züge trägt.

132 Z.B. David Green / Sandra Yip, Fn. 23, S. 20, 28.

133 Bryan Bachner, Fn. 25, S. 229, 248.

134 Bryan Bachner, Fn. 97, S. 635, 646; vgl. auch Dirk Betke, Fn. 4, S. 77.

135

Bryan Bachner, Fn. 25, S. 229, 242 ff.

136 Z.B. Zhou Dadi, Fn. 7, S. 221, 230 (Conclusion). Zur Geschichte dieser Haltung s. Bryan Bachner, Fn. 128. 


\section{ABSTRACTS}

\section{Restructuring Democracy in Haiti}

\section{By Hans-Joachim Heintze}

The international response to the abolition of the democratic government of Haiti may contribute to adopting a middle course between exaggerated expectations of the rule of law on the one hand, and the disappointment about the community of states' unwillingness to pursue international law enforcement on the other. While the community of states hesitated for so long, painful as it was for the people of Haiti suffering under violations of human rights, the applicable international law was elaborated. First, it became clear that there is no obligation under general international law to transform states according to the structures of western democracies. Regional international law is different in some cases. However, when in the case of Haiti the OAS and the UN demanded the return of the elected democratic government, they clearly did not imply obligations under general international law.

Second, the example of Haiti illustrates that even after it has been determined by the U.N. Security Council that an illegal (undemocratic) government constitutes a threat to the peace and international security, it will be extremely difficult to enforce democracy by military force from outside. This political and pragmatic view is supported by international law: The use of force is only permitted in self-defence under Art. 51 of the UN-Charter or in the case of threats to the peace under chapter VII of the UN-Charter.

There is a procedure that should be more frequently applied in future, as it favourably combines the legal with the political side: The higher legitimacy of democratic governments should be utilized more effectively in international relations.

\section{Environmental Law in China}

\section{By Joachim Schabacker}

In China the environmental situation is approaching catastrophic proportions. The Chinese government has responded by setting up an immense environmental protection bureaucracy. They have created a great number of laws and regulations and emended ideology and policy towards "sustainable development", but effectively have accomplished little. The 
basic question is: Why is there so little benefit to be seen since the establishment of this agency:

This article addresses the following issues:

- the environmental situation in China,

- the basic principles of the Chinese environmental law and administration system,

- enforcement problems,

- future policy plans such as incorporating market mechanisms into environmental protection.

The article comes to the conclusion that the key to the future of the environment in China lies in the relationship between economic progress, national security and environmental protection.

\section{Without Truth, No Reconciliation}

\section{By Gerhard Werle}

In June 1995 South Africa passed the Promotion of National Unity and Reconciliation Act. This was South Africa's answer to the problems faced by societies in transition - how shall a new, democratic order deal with the wrongs of its non-democratic past?

The Promotion of National Unity and Reconciliation Act entrusts important prerequisites of reckoning with the past to a Truth and Reconciliation Commission. It divides the Commission into three committees: a Human Rights Committee, an Amnesty Committee and a Committee on Reparation and Rehabilitation. The Human Rights Committee is mandated to investigate serious human rights abuses committed in the course of the conflicts of the past, both by the government and by the liberation movements. It is the task of the Committee to establish the nature, extent and cause of the abuse; its history, the surrounding circumstances and the motives of those involved and the identity of those responsible. Through the Committee on Reparation and Rehabilitation, the Commission is to advise Parliament on the best means of rehabilitating the victims of human rights abuses morally and, to some extent, economically.

The Amnesty Committee deals with one of the most controversial issues in times of transition: whether to punish human rights offenders or grant amnesty for the sake of peace. Here South Africa has adopted a completely new solution by requiring those who need amnesty to apply of it and disclose all relevant facts with their application. In this way the perpetrators themselves contribute to reconciliation by acknowledging the wrongfulness of what they have done and assisting in the investigation. 